\title{
Critical behavior of Tan's contact for bosonic systems with a fixed chemical potential
}

\author{
Abdulla Rakhimov ${ }^{1}$, Tolib Abdurakhmonov ${ }^{1}\left(\mathbb{C}\right.$ and B Tanatar ${ }^{2, *}$ (1) \\ ${ }^{1}$ Institute of Nuclear Physics, Tashkent 100214, Uzbekistan \\ 2 Department of Physics, Bilkent University, Bilkent, 06800 Ankara, Turkey \\ E-mail: tanatar@fen.bilkent.edu.tr
}

Received 3 May 2021, revised 9 August 2021

Accepted for publication 18 August 2021

Published 1 September 2021

\begin{abstract}
The temperature dependence of Tan's contact parameter $C$ and its derivatives for spin gapped quantum magnets are investigated. We use the paradigm of Bose-Einstein condensation (BEC) to describe the low temperature properties of quasiparticles in the system known as triplons. Since the number of particles and the condensate fraction are not fixed we use the $\mu V T$ ensemble to calculate the thermodynamic quantities. The interactions are treated at the Hartree-Fock-Bogoliubov approximation level. We obtained the temperature dependence of $C$ and its derivative with respect to temperature and applied magnetic field both above and below $T_{\mathrm{c}}$ of the phase transition from the normal phase to BEC. We have shown that $C$ is regular, while its derivatives are discontinuous at $T_{\mathrm{c}}$ in accordance with Ehrenfest's classification of phase transitions. Moreover, we have found a sign change in $\partial C / \partial T$ close to the critical temperature. As to the quantum critical point, $C$ and its derivatives are regular as a function of the control parameter $r$, which induces the quantum phase transition. At very low temperatures, one may evaluate $C$ simply from the expression $C=m^{2} \mu^{2} / \bar{a}^{4}$, where the only parameter effective mass of quasiparticles should be estimated. We propose a method for measuring of Tan's contact for spin gapped dimerized magnets.
\end{abstract}

Keywords: Tan's contact, spin gapped quantum magnets, triplons

(Some figures may appear in colour only in the online journal)

\section{Introduction}

Some years ago, Tan derived a set of exact relations that link the short distance large-momentum correlations to the bulk thermodynamic properties of a fermionic system with shortrange inter-particle interactions [1-3]. They are connected by a single coefficient $C$, referred to as the integrated contact intensity or 'contact'. Further, Tan's ideas were developed $[4,5]$ and extended to Bose systems also [6-9]. It has been established that Tan's contact measures the density of pairs at short distances and determines the exact large-momentum or high frequency behavior of various physical observables. It serves

\footnotetext{
* Author to whom any correspondence should be addressed.
}

as an important quantity to characterize strongly interacting many-body systems [10].

Experimentally, Tan's relations have been confirmed both for fermions (especially for quasi-1D systems) [11-13] and Bose gases [14-18].

Although, there are several theoretical studies of contact of low dimensional Fermi systems even at finite temperatures $[4,19-24]$, the temperature dependence of bosonic contact in three dimensions is mostly unknown.

Studying the temperature dependence of contact parameter gives an opportunity to know its critical behavior near phase transitions. For example, Chen et al [25] have shown that contact and its derivatives are uniquely determined by the universality class of the phase transition for fermions in the critical 
region. In a system of bosons, critical behavior of thermodynamic quantities have some specifics due to Bose-Einstein condensation (BEC) at low temperatures. For instance, the specific heat is discontinuous at the critical temperature $T_{\mathrm{c}}$, and the Grüneisen parameter changes its sign [26, 27].

The main goal of the present work is to study the temperature dependence of Tan's contact for bosons, as well as its derivatives, to draw conclusions about their critical behavior near $T_{\mathrm{c}}$ and close to the quantum critical point (QCP) of the quantum phase transition (QPT).

It is well known that physical systems at equilibrium are studied in statistical mechanics through statistical ensembles. For example, a system that the canonical ensemble exchanges only energy with the surroundings is called $N V T$, where $N$ is the number of atoms of a gas in the volume $V$ at temperature $T$. On the other hand, a grand canonical ensemble also makes it possible for particles to exchange, with the parameter $\mu$, which may be interpreted as the chemical potential, and is called $\mu V T$. In the former case, $N$ or $\rho=N / V$ is fixed and the chemical potential is determined by the equation of state, while in the latter case $\mu$ is fixed as an input parameter which defines number of particles through a thermodynamic equation [28]. Tan's relations including the contact have been thoroughly studied for $N V T$ ensembles, however, the investigations of contact for a $\mu V T$ ensemble is still missing. In this work, we choose a system of bosonic quasiparticles introduced in bond operator formalism $[29,30]$ to describe the singlet-triplet excitations in spin gapped magnetic materials [31]. It is well established that low temperature properties of such a class of quantum magnets could be described within the paradigm of BEC of these quasiparticles called triplons. Therefore, one may conclude that, at low temperature, thermodynamic properties of such materials are mainly determined (but not only) by the condensation (and depletion) of triplons [27, 31-33]. Triplon concept has found application in other spin-gapped models as well [34].

There is one more reason of our choice of spin gapped quantum magnets to study Tan's contact. In such systems, the number of particles $N$ and the condensate fraction $N_{0} / N$ can be directly evaluated by measuring the uniform magnetization $M$ and staggered magnetization $M_{\perp}$ respectively, as $N=M / g \mu_{\mathrm{B}}$, $N_{0}^{2}=2 M_{\perp}^{2} /\left(g \mu_{\mathrm{B}}\right)^{2}$ [35], where $g$ is the electron Landé factor and $\mu_{\mathrm{B}}$ is the Bohr magneton. Evidently, the final analytic expression for $C$ will include $M$ and $M_{\perp}$, making the contact parameter to be easily measured.

In practice, Tan's contact for bosons with zero range interaction may be evaluated by any of following Tan's relations [10]. For example,

(a) By the asymptotic behavior of momentum distribution $n_{k}$,

$$
C=\lim _{k \rightarrow \infty} k^{4} n_{k}
$$

(b) By Tan's sweep theorem as

$$
C=\frac{8 \pi m a^{2}}{V} \begin{cases}\left(\frac{\partial F}{\partial a}\right)_{N, T} & \text { for } N V T \text { ensembles } \\ \left(\frac{\partial \Omega}{\partial a}\right)_{\mu, T} & \text { for } \mu V T \text { ensembles }\end{cases}
$$

where $F$ and $\Omega$ are the free energy and the grand canonical potential, respectively [36]; $a$ is the scattering length and $m$ is the effective mass of the particle.

(c) By using Hellman-Feynman theorem [4]

$$
C=\frac{16 \pi^{2} a^{2}}{V} \int \mathrm{d} \vec{r}\left\langle\psi^{\dagger}(\vec{r}) \psi^{\dagger}(\vec{r}) \psi(\vec{r}) \psi(\vec{r})\right\rangle .
$$

In our previous paper [37], it has been shown that if one uses mean field theory (MFT), evaluation of $C$ from equation (1.2) will be the most convenient and reliable. Consequently, we study $C$ of a triplon gas at finite temperature in the framework of MFT, namely in the Hartree-Fock-Bogoliubov (HFB) approximation [38]. We stress here that below we shall discuss $C$ only for homogeneous systems. For inhomogeneous systems, especially at very low temperatures, one may also use the Gross-Pitaevskii equation to study the dynamics of the condensate $[39,40]$ as well as that of the Tan's contact.

For convenience, we adopt units such that $\hbar=1, k_{\mathrm{B}}=1$ and $V=1$ in the following text. In these units $C$ is dimensionless. In natural units $C$ may be obtained further by dividing it by $\bar{a}^{4}$, getting it in (length) $)^{-4}$ where $\bar{a}$ is the average lattice parameter, $\bar{a}=V^{1 / 3}$, and $V$ is the unit cell volume of the crystal.

This article is organized as follows. In section 2, we outline main equations for a triplon gas at finite temperature in the HFB approximation, which will be used to evaluate the Tan's contact and its derivatives in section 3 . Our discussions and conclusions will be presented in section 4 .

\section{Triplon density in the HFB approximation}

We start with the effective Hamiltonian of triplons as a nonideal Bose gas with contact repulsive interaction

$$
\hat{H}=\int \mathrm{d} \vec{r}\left[\psi^{\dagger}(\vec{r})(\hat{K}-\mu) \psi(\vec{r})+\frac{U}{2}\left(\psi^{\dagger}(\vec{r}) \psi(\vec{r})\right)^{2}\right],
$$

where $\psi(\vec{r})$ is the bosonic field operator, $U=4 \pi a / m$ is the interaction strength, and $\hat{K}$ is the kinetic energy operator which defines the bare triplon dispersion $\varepsilon_{\vec{k}}$ in momentum space. Since the triplon BEC occurs in solids, the integration is performed over the unit cell of the crystal with the corresponding momenta defined in the first Brillouin zone [41]. The parameter $\mu$ characterizes an additional direct contribution to the triplon energy due to the external field

$$
\mu=g \mu_{\mathrm{B}} H-\Delta
$$

and it can be interpreted as the chemical potential of the $S_{z}=-1$ triplons [42]. In equation (2.2), $\Delta=g \mu_{\mathrm{B}} H_{\mathrm{c}}$, is the spin gap separating the ground-state from the lowest energy triplet excitation and $H_{\mathrm{c}}$ is the critical external magnetic field [43]. The effective Hamiltonian $\hat{H}$ has the following free parameters characterizing a given material: $g, H_{\mathrm{c}}, U$ and possibly, an effective triplon mass $m$ for the case of a simple bare dispersion given as $\varepsilon_{\vec{k}}=\vec{k}^{2} / 2 m .^{3}$

\footnotetext{
${ }^{3}$ In general, a realistic $\varepsilon_{\vec{k}}$ includes several other parameters [44].
} 
In general the Hamiltonian in equation (2.1) is invariant under global $U(1)$ gauge transformation $\psi(\vec{r}) \rightarrow \mathrm{e}^{\mathrm{i} \alpha} \psi(\vec{r})$ with a real number $\alpha$. However, this symmetry is broken in the condensate phase, where $T<T_{\mathrm{c}}$, and it is restored in the normal phase, $T \geqslant T_{\mathrm{c}}$.

This transition temperature $T_{\mathrm{c}}$, which corresponds to the vanishing of the condensate density $\rho_{0}\left(T_{\mathrm{c}}\right)=0$ may be calculated from the following equation

$$
\sum_{k} \frac{1}{\mathrm{e}^{\varepsilon_{k} / T_{\mathrm{c}}}-1}=\frac{\mu}{2 U} \equiv \rho_{\mathrm{c}}
$$

Note that for existing quantum magnets with a spin gap $T_{\mathrm{c}}$ is rather large, $T_{\mathrm{c}} \approx 2 \mathrm{~K}$, in contrast to the critical temperature of BEC in atomic gases, where the number of particles is fixed and the critical temperature is of the order of nanokelvins. However, in the $\mu V T$ ensemble under discussion, the particle density depends on the external magnetic field through the chemical potential $\mu$ as

$$
\rho\left(T \geqslant T_{\mathrm{c}}\right)=\sum_{k} \frac{1}{\mathrm{e}^{\beta \omega_{k}}-1}, \quad \omega_{k}=\varepsilon_{k}-\mu+2 U \rho,
$$

in the normal phase. In the BEC phase, an explicit expression for $\rho\left(T<T_{\mathrm{c}}\right)$ strongly depends on the chosen version of an approximation. For example, in the HFB approximation, which is employed here, the particle density and condensate fraction are given by the following set of equations [45]

$$
\begin{aligned}
& \rho\left(T<T_{\mathrm{c}}\right)=\rho_{0}+\rho_{1}=\frac{\mu+\mu_{\mathrm{eff}}}{2 U}, \\
& \mu_{\mathrm{eff}}=\mu+2 U\left(\sigma-\rho_{1}\right), \\
& \rho_{1}=\frac{1}{2} \sum_{k}\left[\frac{\operatorname{coth}\left(\beta E_{k} / 2\right)\left(\varepsilon_{k}+\mu_{\mathrm{eff}}\right)}{E_{k}}-1\right], \\
& \sigma=-\frac{\mu_{\mathrm{eff}}}{2} \sum_{k} \frac{\operatorname{coth}\left(\beta E_{k} / 2\right)}{E_{k}},
\end{aligned}
$$

where $\rho_{1}$ and $\sigma$ correspond to the density of non-condensed particles and anomalous density, respectively. The hyperbolic function in equation (2.5) can be represented also as $\operatorname{coth}(\beta x / 2)=1 / 2+f_{\mathrm{B}}(x)$, where $f_{\mathrm{B}}(x)=1 /(\exp (\beta x)-1)$ is the Bose distribution function.

Note that neglecting $\sigma$ leads to an unexpected cusp in magnetization [46]. In equation (2.5), $E_{k}$ is the dispersion of quasiparticles (bogolons) given as $E_{k}=\sqrt{\varepsilon_{k}} \sqrt{\varepsilon_{k}+2 \mu_{\text {eff }}}$ with the speed of sound $c=\sqrt{\mu_{\mathrm{eff}} / m}$, where $m$ has the meaning of the triplon effective mass, corresponding to the limit of small momenta $\varepsilon_{k} \approx \vec{k}^{2} / 2 m$. The typical value of $m$, used in the literature, [31] is rather small, $m \approx 0.02 \mathrm{~K}$.

\section{Tan's contact for a triplon gas}

As it is pointed out in the introduction, for the $\mu V T$ ensemble it is convenient to calculate $C$ using

$$
C=2 U^{2} m^{2}\left(\frac{\partial \Omega}{\partial U}\right)_{T, \mu},
$$

where the grand thermodynamic potential $\Omega$ has the following total derivative [10, 32, 33]

$$
\mathrm{d} \Omega=-S \mathrm{~d} T-P \mathrm{~d} V-N \mathrm{~d} \mu-M \mathrm{~d} H+\frac{C \mathrm{~d} a}{8 \pi m a^{2}},
$$

in which $S$ is the entropy and $P$ is the pressure. Note that the relation between the magnetization $M$ and the triplon density may be directly obtained from equation (3.2) as $M=g \mu_{\mathrm{B}} \rho$. Moreover, using equation (3.2) one may find useful expressions for the derivatives of $C$ as

$$
\begin{aligned}
\left(\frac{\partial C}{\partial T}\right) & =-2 U^{2} m^{2}\left(\frac{\partial S}{\partial U}\right),\left(\frac{\partial C}{\partial \mu}\right) \\
& =\frac{1}{g \mu_{\mathrm{B}}}\left(\frac{\partial C}{\partial H}\right)=-2 U^{2} m^{2}\left(\frac{\partial \rho}{\partial U}\right) .
\end{aligned}
$$

It is well known that from BEC to a normal phase transition is a second order phase transition, in which the entropy $S(T, H, a)$ is continuous across the critical temperature i.e.

$$
\begin{aligned}
\mathrm{d} S\left(T=T_{\mathrm{c}}^{-}\right)= & \mathrm{d} S\left(T=T_{\mathrm{c}}^{+}\right)=\left(\frac{\partial S}{\partial T}\right)_{H, a} \mathrm{~d} T \\
& +\left(\frac{\partial S}{\partial H}\right)_{T, a} \mathrm{~d} H+\left(\frac{\partial S}{\partial a}\right)_{H, T} \mathrm{~d} a .
\end{aligned}
$$

Therefore, using equations (3.2)-(3.4) leads to an extended Ehrenfest relation:

$$
\begin{aligned}
T_{\mathrm{c}}^{-1} \Delta\left[C_{H}\right]= & -\Delta\left[\left(\frac{\partial M}{\partial T}\right)_{H, U}\right]\left(\frac{\mathrm{d} H}{\mathrm{~d} T}\right) \\
& +\frac{1}{2 U^{2} m^{2}} \Delta\left[\left(\frac{\partial C}{\partial T}\right)_{H, U}\right]\left(\frac{\mathrm{d} U}{\mathrm{~d} T}\right),
\end{aligned}
$$

where $C_{H}=T(\partial S / \partial T)$ is the heat capacity and $\Delta[f] \equiv$ $f\left(T=T_{\mathrm{c}}^{-}\right)-f\left(T=T_{\mathrm{c}}^{+}\right)$is the jump in the function $f$ at $T=T_{\mathrm{c}}{ }^{4}$

In the following we discuss the normal and BEC phases, separately.

\subsection{Normal phase $\left(T \geqslant T_{\mathrm{c}}\right)$}

In the normal phase $\Omega$ is given by

$$
\Omega\left(T \geqslant T_{\mathrm{c}}\right)=-U \rho^{2}+T \sum_{k} \ln \left(1-\mathrm{e}^{\beta \omega_{k}}\right),
$$

where $\rho$ and $\omega_{k}$ are defined in equation (2.4). Now, taking the derivative of $\Omega$ and using equation (2.4) one may easily find

$$
\left(\frac{\partial \Omega}{\partial U}\right)_{T \geqslant T_{\mathrm{c}}}=-\rho^{2}-2 U \rho \rho^{\prime}+\left(2 \rho+2 U \rho^{\prime}\right) \sum_{k} \frac{1}{\mathrm{e}^{\beta \omega_{k}}-1}=\rho^{2},
$$

\footnotetext{
${ }^{4}$ A similar relation for $N V T$ ensembles will be $T_{\mathrm{c}}^{-1} \Delta\left[C_{P}\right]=-\Delta$ $\left[\left(\frac{\partial P}{\partial T}\right)_{V, U}\right]\left(\frac{\mathrm{d} V}{\mathrm{~d} T}\right)+\frac{1}{2 U^{2} m^{2}} \Delta\left[\left(\frac{\partial C}{\partial T}\right)_{V, U}\right]\left(\frac{\mathrm{d} U}{\mathrm{~d} T}\right)$ when the entropy is considered as a function of temperature, volume and the strength of the contact interaction.
} 
and hence

$$
C\left(T \geqslant T_{\mathrm{c}}\right)=2 U^{2} m^{2} \rho^{2}=\frac{2 U^{2} m^{2} M^{2}}{\left(g \mu_{\mathrm{B}}\right)^{2}} .
$$

The derivatives of $C$ can be evaluated using equation (3.3) or directly from equation (3.8) as

$$
\begin{aligned}
& \left(\frac{\partial C}{\partial T}\right)_{T \geqslant T_{\mathrm{c}}}=4 U^{2} m^{2} \rho\left(\frac{\partial \rho}{\partial T}\right)=-2 m^{2} U^{2}\left(\frac{\partial S}{\partial U}\right), \\
& \left(\frac{\partial C}{\partial \mu}\right)_{T \geqslant T_{\mathrm{c}}}=4 U^{2} m^{2} \rho\left(\frac{\partial \rho}{\partial \mu}\right)=-2 m^{2} U^{2}\left(\frac{\partial \rho}{\partial U}\right),
\end{aligned}
$$

where [27]

$$
\left(\frac{\partial S}{\partial U}\right)_{T \geqslant T_{\mathrm{c}}}=\frac{2 \rho \beta I_{1}}{1-2 I_{2}}, \quad\left(\frac{\partial \rho}{\partial U}\right)_{T \geqslant T_{\mathrm{c}}}=\frac{2 \rho I_{2}}{U\left[1-2 I_{2}\right]},
$$

and the integrals $I_{1}$ are $I_{2}$ are given in the appendix A. Note that equation (3.9) lead to the following relations for the susceptibility, $\chi=(\partial M / \partial H)$ in the normal phase

$$
\begin{aligned}
\left(\frac{\partial C}{\partial H}\right)_{T \geqslant T_{\mathrm{c}}} & =\frac{4 U^{2} m^{2}}{\left(g \mu_{\mathrm{B}}\right)^{2}}(M \chi)_{T \geqslant T_{\mathrm{c}}}, \chi\left(T \geqslant T_{\mathrm{c}}\right) \\
& =-\frac{\left(g \mu_{\mathrm{B}}\right)^{2}}{2 M}\left(\frac{\partial M}{\partial U}\right)_{T \geqslant T_{\mathrm{c}}} .
\end{aligned}
$$

\subsection{Condensed phase $\left(T<T_{\mathrm{c}}\right)$}

In the condensed phase the thermodynamic potential is given by $[30,32,33]$

$$
\begin{aligned}
\Omega\left(T<T_{\mathrm{c}}\right)= & \frac{U \rho_{0}^{2}}{2}-\mu \rho_{0}-\frac{U}{2}\left(2 \rho_{1}^{2}+\sigma^{2}\right) \\
& +\frac{1}{2} \sum_{k}\left(E_{k}-\varepsilon_{k}-\mu_{\mathrm{eff}}\right)+T \sum_{k} \ln \left(1-\mathrm{e}^{-\beta E_{k}}\right)
\end{aligned}
$$

with $\mu_{\text {eff }}, \rho_{1}$ and $\rho$ are given by equation (2.5), $\rho_{0}=\rho$ $-\rho_{1}=\mu_{\mathrm{eff}} / U-\sigma$ and $E_{k}=\sqrt{\varepsilon_{k}} \sqrt{\varepsilon_{k}+2 \mu_{\mathrm{eff}}}$. For simplicity, one may start with the well-known Bogoliubov approximation, where the depletion and anomalous density are completely neglected. For atomic gases, this leads to the Gross-Pitaevskii equation which is successfully used in a lot of systems [10]. In the present case Bogoliubov approximation corresponds to $\rho_{0} \approx \rho, \mu_{\mathrm{eff}} \approx \mu=U \rho$ and hence

$$
\begin{aligned}
\Omega_{\text {Bogoliubov }}\left(T<T_{\mathrm{c}}\right)= & -\frac{\mu^{2}}{2 U}+\frac{1}{2} \sum_{k}\left[E_{k}-\varepsilon_{k}-\mu\right] \\
& +T \sum_{k} \ln \left(1-\mathrm{e}^{-E_{k} \beta}\right) .
\end{aligned}
$$

Since in $\mu V T$ ensembles the chemical potential does not depend on the inter-particle interaction, i.e. $(\partial \mu / \partial U)=0$, one
Table 1. Material parameters used in our numerical calculations. The parameters $g, H_{\mathrm{c}}, \bar{a}$ and $\Delta$ are taken directly from the experimental measurements, while $m$ and $U$ are optimized to fit experimental magnetizations (see reference [27] for details).

\begin{tabular}{lcccccr}
\hline & $g$ & $H_{\mathrm{c}}[\mathrm{T}]$ & $m\left[\mathrm{~K}^{-1}\right]$ & $U[\mathrm{~K}]$ & $\Delta[\mathrm{K}]$ & $\bar{a}[\AA]$ \\
\hline $\mathrm{Ba}_{3} \mathrm{Cr}_{2} \mathrm{O}_{8}$ & 1.95 & 12.10 & 0.2 & 20 & 15.85 & 3.97 \\
$\mathrm{Sr}_{3} \mathrm{Cr}_{2} \mathrm{O}_{8}$ & 1.95 & 30.40 & 0.06 & 51.2 & 39.8 & 3.82 \\
$\mathrm{TlCuCl}_{3}$ & 2.06 & 5.1 & 0.02 & 315 & 7.1 & 7.93 \\
\hline
\end{tabular}

immediately obtains from equation (3.13) Tan's contact in the Bogoliubov approximation as ${ }^{5}$

$$
C_{0}=m^{2} \mu^{2}
$$

From this equation, it is seen that in this simple approximation Tan's contact does not depend on temperature, which means that in $\mu V T$ ensembles Bogoliubov approximation is not applicable to finite temperatures. The quality of this approximation at $T=0$ is tested below. In figure 1, Tan's contact as a function of the magnetic field is presented for two different compounds $\mathrm{TlCuCl}_{3}$ (figure 1(a)) and $\mathrm{Ba}_{3} \mathrm{Cr}_{2} \mathrm{O}_{8}$ (figure 1(b)) in natural units, i.e. in $\AA^{-4}$. It is seen that for both compounds, the difference between the exact HFB (solid lines) and simple Bogoliubov approximation (dashed lines) is rather small, especially for small chemical potentials. Remarkably, the contact given by equation (3.14) does not include the scattering length of triplon interaction but includes only the effective mass as an internal microscopic parameter of the system. This is why the contact for $\mathrm{TlCuCl}_{3}$ at the same magnetic field e.g. at $H=13.5$ $\mathrm{T}$, is much smaller than that for $\mathrm{Ba}_{3} \mathrm{Cr}_{2} \mathrm{O}_{8}$. In fact, as it is seen from table 1 the ratio of effective masses is of the order of $m\left(\mathrm{Ba}_{3} \mathrm{Cr}_{2} \mathrm{O}_{8}\right) / m\left(\mathrm{TlCuCl}_{3}\right) \approx 10$.

Although $C_{0}$ in equation (3.14) is not good at finite temperatures, it may serve as a characteristic scale for the contact $C$. Thus, differentiating $\Omega$ in equation (3.12) with respect to $U$ we obtain the following explicit expression for the contact in HFB approximation

$$
\begin{aligned}
C\left(T<T_{\mathrm{c}}\right)= & C_{0}\left\{\tilde{\rho}-\frac{\tilde{\rho}_{1}^{2}+\tilde{\rho}^{2}-\tilde{\sigma}^{2}}{4}+2 \rho_{\mathrm{c}} \mu_{\mathrm{eff}}^{\prime}\left[I_{3}-1-U \tilde{\sigma} \sigma^{\prime}\right.\right. \\
& \left.\left.+\frac{\tilde{\rho}_{0}}{2}+U \rho_{1}^{\prime}\left(2-\tilde{\rho}_{1}-\tilde{\rho}\right)\right]\right\}
\end{aligned}
$$

where $\tilde{\rho}=\rho / \rho_{\mathrm{c}}, \quad \tilde{\rho}_{1}=\rho_{1} / \rho_{\mathrm{c}}, \quad \tilde{\rho}_{0}=\rho_{0} / \rho_{\mathrm{c}}, \quad \tilde{\sigma}=\sigma / \rho_{\mathrm{c}}, \rho_{1}^{\prime}=$ $\left(\partial \rho_{1} / \partial \mu_{\mathrm{eff}}\right), \sigma^{\prime}=\left(\partial \sigma / \partial \mu_{\mathrm{eff}}\right), \mu_{\text {eff }}^{\prime}=\left(\partial \mu_{\mathrm{eff}} / \partial U\right)=\left(\tilde{\sigma}-\tilde{\rho}_{1}\right) /$ $\left[1+2 U\left(\rho_{1}^{\prime}-\sigma^{\prime}\right)\right]$ and $\rho_{\mathrm{c}}=\mu / 2 U$. The explicit expressions for $I_{3}, \rho_{1}^{\prime}$ and $\sigma^{\prime}$ are given in the appendix A. Note that, due to the factoring out of $C_{0}$, the quantity in curly brackets is dimensionless.

Now, using equations (3.3) and (3.12), yields ${ }^{5}$ In natural units $C_{0}=m^{2} \mu^{2} / \bar{a}^{4}$, where the average lattice parameter $\bar{a}$ can be
taken from table 1 . 


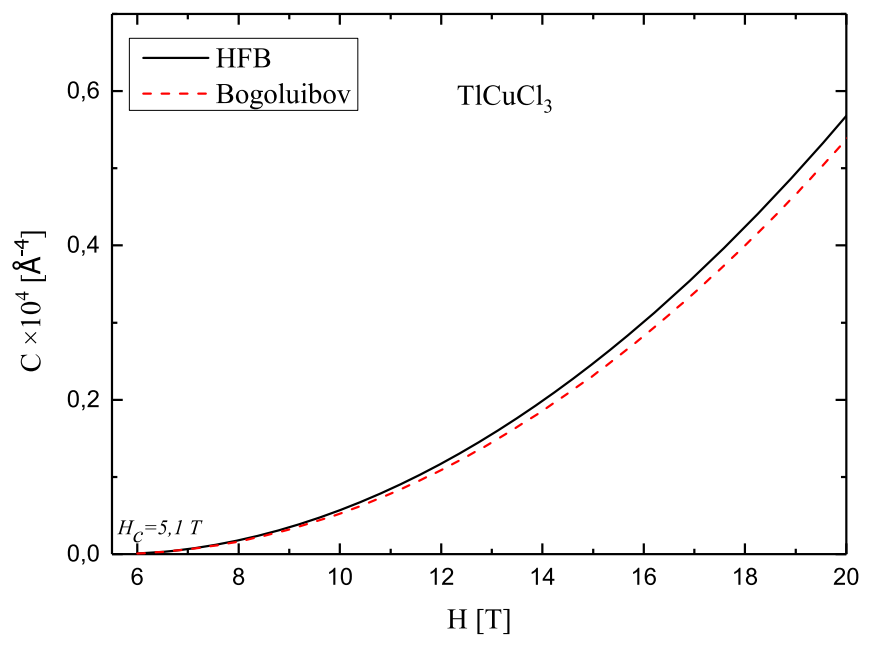

a)

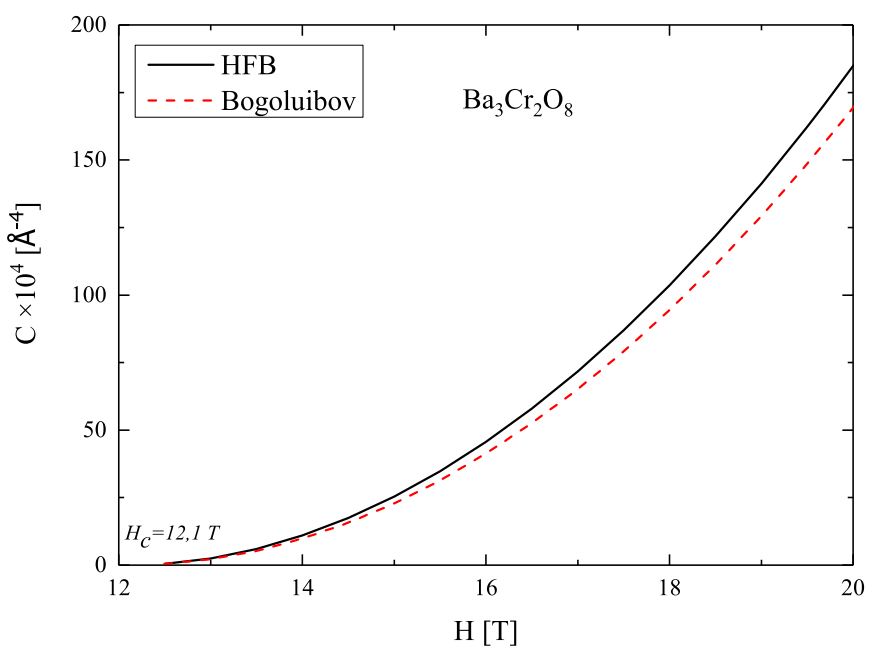

b)

Figure 1. Tan's contact at zero temperature for $\mathrm{TlCuCl}_{3}$ (a) and $\mathrm{Ba}_{3} \mathrm{Cr}_{2} \mathrm{O}_{8}$ (b) in $\AA^{-4}$ in $\mathrm{HFB}$ (solid lines) and Bogoliubov approximations (dashed lines). The input parameters are given in table 1 .

$$
\begin{aligned}
S\left(T<T_{\mathrm{c}}\right)= & -\sum_{k} \ln \left(1-\mathrm{e}^{-\beta E_{k}}\right) \\
& +\beta \sum_{k} E_{k} f_{\mathrm{B}}\left(E_{k}\right),\left(\frac{\partial C}{\partial T}\right)_{T<T_{\mathrm{c}}} \\
= & -2 m^{2} U^{2}\left(\frac{\partial S}{\partial U}\right) \\
= & 8 \beta\left(U m \rho_{\mathrm{c}}\right)^{2} \rho_{\mathrm{c}} I_{4} \mu_{\mathrm{eff}}^{\prime},\left(\frac{\partial C}{\partial H}\right)_{T<T_{\mathrm{c}}} \\
= & \frac{1}{g \mu_{\mathrm{B}}}\left(\frac{\partial C}{\partial \mu}\right)=-\frac{2 m^{2} U^{2}}{g \mu_{\mathrm{B}}}\left(\frac{\partial \rho}{\partial U}\right) \\
= & \frac{m^{2} \mu\left(\tilde{\rho}-2 \rho_{\mathrm{c}} \mu_{\mathrm{eff}}^{\prime}\right)}{g \mu_{\mathrm{B}}}=-\frac{2 m^{2} U^{2}}{\left(g \mu_{\mathrm{B}}\right)^{2}}\left(\frac{\partial M}{\partial U}\right) .
\end{aligned}
$$

Unlike in the normal phase, for $T<T_{\mathrm{c}}$ we failed to find a simple relation between susceptibility and $(\partial C / \partial \mu)$ similar to equation (3.11).

In the previous subsection we have shown that at zero temperature the contact may be conveniently approximated as $C(T=0) \approx C_{0}=m^{2} \mu^{2}$. We now analyze the temperature dependence of $C\left(T<T_{\mathrm{c}}\right)$ given in equation (3.15) which requires solving the set of equations (2.5). In figure 2(a), we present $C(T)$ for three compounds $\mathrm{Ba}_{3} \mathrm{Cr}_{2} \mathrm{O}_{8}, \mathrm{Sr}_{3} \mathrm{Cr}_{2} \mathrm{O}_{8}$, and $\mathrm{TlCuCl}_{3}$ calculated in the HFB approximation (solid curves) using equations (3.8) and (3.15). It is seen that contact at finite temperature can be at least qualitatively approximated as

$$
C(T) \approx 2 U^{2} m^{2} M^{2} /\left(g \mu_{\mathrm{B}}\right)^{2},
$$

(dashed lines) which coincides with the exact one in the normal phase. In figure 2(b) the magnetizations for the same compounds as a function of temperature are also presented for completeness.

\subsection{Contact and its derivatives near critical temperature}

The crude approximation in equation (3.17) is also useful to explain the temperature dependence of $(\partial C / \partial H)$ and $(\partial C / \partial T)$ presented in figures 3(a) and (b), respectively. In fact, it follows that

$$
\begin{aligned}
\left(\frac{\partial C}{\partial H}\right) & \propto\left(\frac{\partial M^{2}}{\partial H}\right) \propto M \chi,\left(\frac{\partial C}{\partial T}\right) \\
& \propto\left(\frac{\partial M^{2}}{\partial T}\right)=2 M\left(\frac{\partial M}{\partial T}\right) .
\end{aligned}
$$

The experimental susceptibilities of quantum magnets with a spin gap show a well-pronounced maximum at $T=T_{\mathrm{c}}$, and they decrease exponentially at low temperatures indicating the existence of a gap $\Delta$ between the ground-state and first excited triplet states $[35,47,48]$, while the magnetization has a local minimumi.e. $\left.(\partial M / \partial T)\right|_{T=T_{\mathrm{c}}}=0$ at the critical point (see figure 2(b)). This is in good agreement with our predicted temperature dependence of derivatives of the contact: $(\partial C / \partial H)$ has a maximum and $(\partial C / \partial T)$ changes its sign near the phase transition from BEC to a normal phase.

On the other hand, it is well established that the heat capacity $C_{V}$ has a cusp near the transition point [49-52]. Therefore, we ask whether the quantities under consideration have a jump near $T_{\mathrm{c}}$. To study this question we consider equations (3.8) and (3.15). From equation (3.8) one may see that $C\left(T \rightarrow T_{\mathrm{c}}^{+}\right)=2 U^{2} m^{2} \rho_{\mathrm{c}}^{2}$. On the other side, setting in (3.15) $\tilde{\rho}_{1}=\tilde{\rho}=1, \sigma=0, \mu_{\text {eff }}=0$ and $E_{k}=\varepsilon_{k}$, one obtains $C\left(T \rightarrow T_{\mathrm{c}}^{-}\right)=m^{2} \mu^{2}\left[1 / 2+\left(I_{3}-1\right) 2 \rho_{\mathrm{c}} \mu_{\mathrm{eff}}^{\prime}\right]=2$ $U^{2} m^{2} \rho_{\mathrm{c}}^{2}$, where we used $I_{3}\left(T=T_{\mathrm{c}}\right)=\sum_{k} f_{\mathrm{B}}\left(\varepsilon_{k}\right)=\rho_{\mathrm{c}}$ and $\mu=2 U \rho_{\mathrm{c}}$. Therefore, Tan's contact is continuous at the critical temperature: $C\left(T \rightarrow T_{\mathrm{c}}^{-}\right)=C\left(T \rightarrow T_{\mathrm{c}}^{+}\right)=2\left(U m \rho_{\mathrm{c}}\right)^{2}$. As to its derivatives, using explicit expression for $(\partial C / \partial \mu)$ and $(\partial C / \partial T)$ given by equations (3.9) and (3.16), it can be demonstrated that they have a cusp at $T=T_{\mathrm{c}}$. Presenting the details in the appendix A, we bring here the final expression for the discontinuities in dimensionless units 


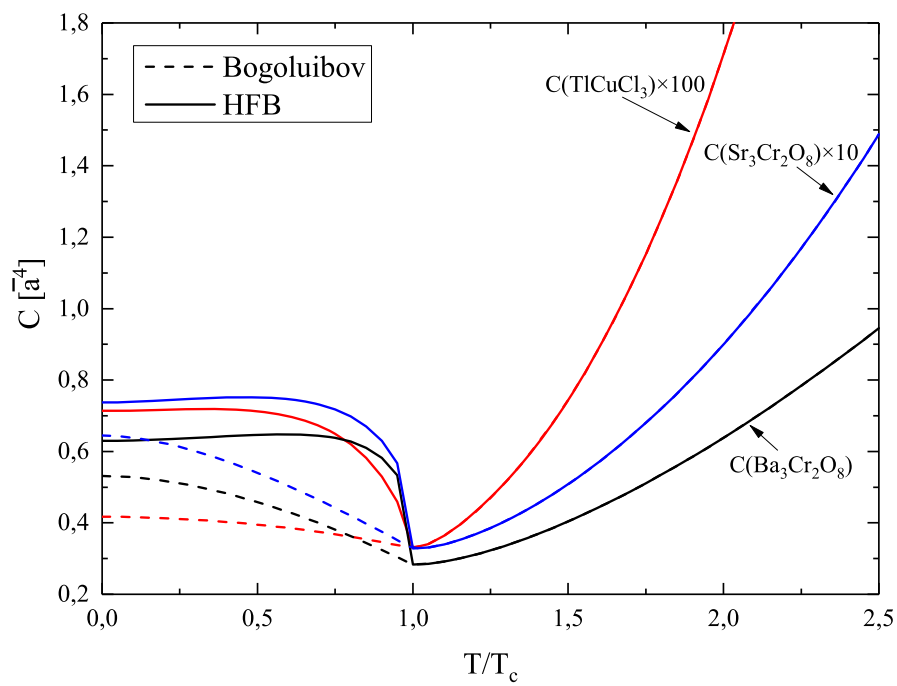

a)

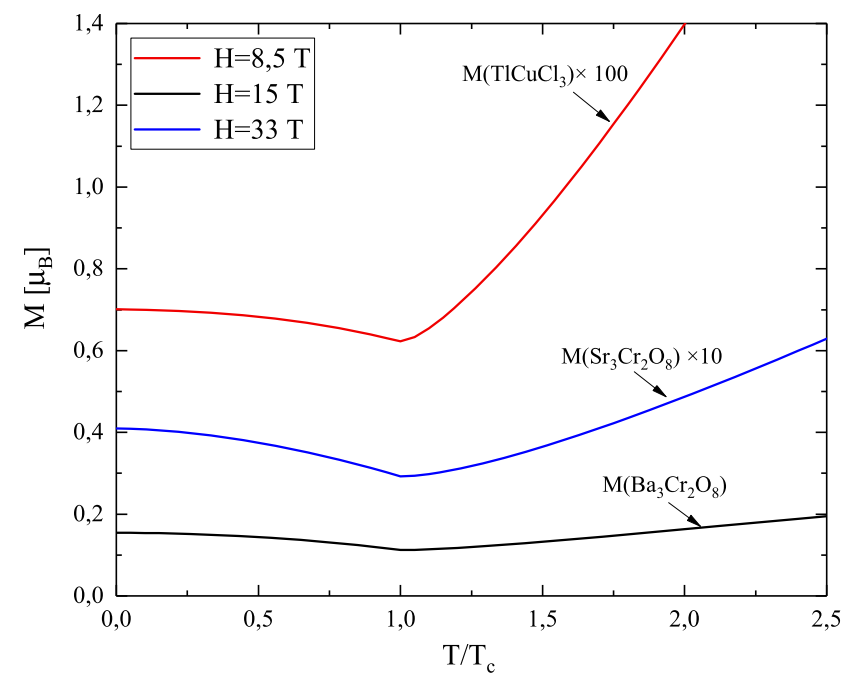

b)

Figure 2. (a) Contact for spin gapped magnets in dimensionless units. The solid lines are for HFB approximation. The dashed lines correspond to the classic approximation given in equation (3.17); (b) the magnetizations $M(T)$ per dimer.

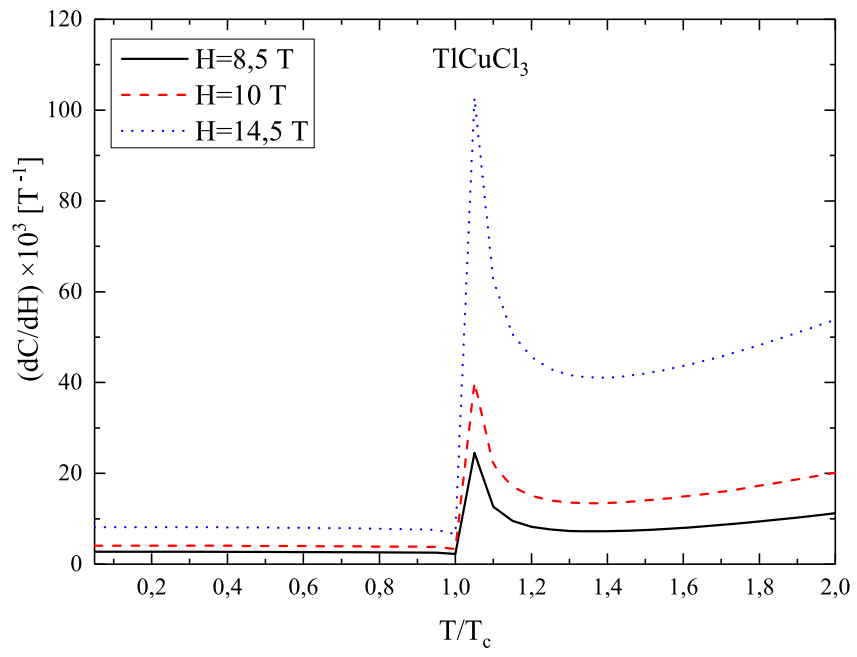

a)

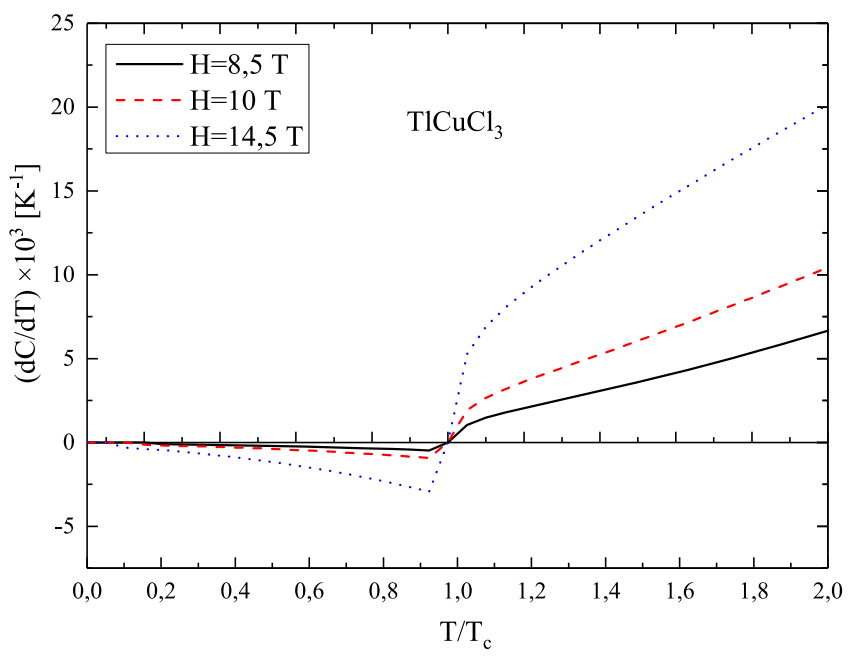

b)

Figure 3. Derivatives of contact with respect to magnetic field (a) and to temperature for $\mathrm{TlCuCl}_{3}$. Here we use $V=1$ units, so $(\partial C / \partial H)$ and $(\partial C / \partial T)$ are given in $\mathrm{T}^{-1}$ and $\mathrm{K}^{-1}$, respectively. The curves are obtained from equations (3.16).

$$
\begin{aligned}
R_{H} & \equiv \frac{H_{\mathrm{c}}}{C_{0}}\left\{\left(\frac{\partial C}{\partial H}\right)_{T=T_{\mathrm{c}}-0}-\left(\frac{\partial C}{\partial H}\right)_{T=T_{\mathrm{c}}+0}\right\} \\
& =\frac{4 T_{\mathrm{c}}}{U}\left\{\sum_{k}\left[1+\frac{4 f_{\mathrm{B}}\left(\varepsilon_{k}\right) T_{\mathrm{c}}}{\varepsilon_{k}}-4 f_{\mathrm{B}}^{2}\left(\varepsilon_{k}\right)\right]\right\}^{-1}, \\
R_{T} & =\frac{T_{\mathrm{c}}}{C_{0}}\left\{\left(\frac{\partial C}{\partial T}\right)_{T=T_{\mathrm{c}}-0}-\left(\frac{\partial C}{\partial T}\right)_{T=T_{\mathrm{c}}+0}\right\} \\
& =-\frac{R_{H}}{\rho_{\mathrm{c}} T_{\mathrm{c}}} \sum_{k} \varepsilon_{k} \mathrm{e}^{\beta \varepsilon_{k}} f_{\mathrm{B}}\left(\varepsilon_{k}\right) .
\end{aligned}
$$

The numerical values of discontinuities $R_{H}$ and $R_{T}$ for $\mathrm{TlCuCl}_{3}$ for some values of the magnetic field are presented in table 2. From table 2 it may be concluded that the jump in $(\partial C / \partial T) \propto R_{T}$ is negative $\left(R_{T}<0\right)$, in contrast to the jump in $(\partial C / \partial \mu) \propto R_{\mu}>0$, and in the heat capacity as well as in the Grüneisen parameter [27]. Thus, from the relation $R_{T} \approx$ $-\left.2 m^{2} U^{2}(\partial S / \partial U)\right|_{T=T_{\mathrm{c}}}$ one may conclude that, in $\mu V T$ ensembles entropy increases with increasing repulsive interaction strength, i.e. $(\partial S / \partial U)>0$. Next, we discuss another critical phenomenon, which is related to the low temperature critical behavior.

\subsection{Low temperature expansion and QPT}

In a $\mu V T$ ensemble, studied in the present work, a QPT occurs at zero temperature upon tuning the external magnetic field or equivalently the chemical potential. At $T=0$ the distance to 
Table 2. Some critical parameters of $\mathrm{TlCuCl}_{3}$.

\begin{tabular}{lcclcc}
\hline$H[\mathrm{~T}]$ & $T_{\mathrm{c}}[\mathrm{K}]$ & $\rho_{\mathrm{c}}$ & $C\left(T=T_{\mathrm{c}}\right)\left[\mathrm{nm}^{-4}\right]$ & $R_{T}$ & $R_{\mu}$ \\
\hline 6.0 & 2.15 & 0.00120 & 0.000384 & -0.0245 & 0.0134 \\
6.5 & 3.06 & 0.00230 & 0.00140 & -0.0365 & 0.0190 \\
7.0 & 3.73 & 0.00340 & 0.00307 & -0.0458 & 0.0230 \\
7.5 & 4.28 & 0.00450 & 0.00537 & -0.0540 & 0.0263 \\
8.0 & 4.75 & 0.00559 & 0.00831 & -0.0609 & 0.0290 \\
8.5 & 5.16 & 0.00669 & 0.0119 & -0.0671 & 0.0314 \\
9.0 & 5.54 & 0.00779 & 0.0161 & -0.0727 & 0.0336 \\
9.5 & 5.88 & 0.00889 & 0.0210 & -0.0779 & 0.0355 \\
10.0 & 6.20 & 0.00999 & 0.0265 & -0.0826 & 0.0373 \\
10.5 & 6.49 & 0.0111 & 0.0326 & -0.0870 & 0.0390 \\
11.0 & 6.77 & 0.0122 & 0.0394 & -0.0911 & 0.0405 \\
11.5 & 7.04 & 0.0133 & 0.0468 & -0.0950 & 0.0420 \\
12.0 & 7.28 & 0.0144 & 0.0549 & -0.0986 & 0.0420 \\
\hline
\end{tabular}

the QCP is determined by a control parameter, $r(H)$. Near the QCP the control parameter can be linearized around the critical magnetic field $H_{\mathrm{c}}$ as $r(H)=\left(H-H_{\mathrm{c}}\right) / H_{\mathrm{c}}$, which corresponds to $\mu=r \Delta$ (where $\Delta=g \mu_{\mathrm{B}} H_{\mathrm{c}}$ is the spin gap). It is expected that some thermodynamic quantities will diverge at QPT. For example, the Grüneisen parameter which plays an important role in magnetocaloric effect diverges as $\left.\Gamma_{H}\right|_{r \rightarrow 0} \sim 1 / r$ $[26,53]$. In the following we address the critical behavior of Tan's contact and its derivatives close to QCP.

The case of $C$ and $(\partial C / \partial \mu)$ is rather straightforward. Actually, in section 2 , we have shown that at $T=0$ the contact can be simply approximated as $\left.C\right|_{T=0}=m^{2} \mu^{2}$, especially at weak magnetic fields, i.e. as $r \rightarrow 0$ (see figure 1). Thus, one directly obtains

$$
\begin{aligned}
\left.C(T=0)\right|_{r \rightarrow 0} & =m^{2} r^{2} \Delta^{2}+O\left(r^{5 / 2}\right),\left.\left(\frac{\partial C}{\partial \mu}\right)\right|_{T=0, r \rightarrow 0} \\
& =2 m^{2} r \Delta+O\left(r^{3 / 2}\right),
\end{aligned}
$$

to show that in contrast to $\Gamma_{H}$, contact and its derivative $(\partial C / \partial H)$ are regular near QCP.

The case of $(\partial C / \partial T)$ is a little more complicated. Here, one has to find the low temperature expansion for $(\partial S / \partial U)$, as well as small $r$ expansion for the effective chemical potential $\mu_{\mathrm{eff}}(r)$. This leads to following expressions [27]

$$
\begin{aligned}
\left(\frac{\partial S}{\partial U}\right) & =-\mu_{\mathrm{eff}}^{\prime} \beta^{2} \sum_{k} \varepsilon_{k} \mathrm{e}^{\beta E_{k}} f_{\mathrm{B}}^{2}\left(E_{k}\right) \\
& =-\frac{\mu_{\mathrm{eff}}^{\prime} \pi^{2} T^{3}}{15 m s_{0}^{5}}+O\left((T m)^{5}\right), \quad \mu_{\mathrm{eff}}^{\prime}=\left(\frac{\partial \mu}{\partial U}\right) \\
& =-\frac{r Q_{0} m \Delta_{1}}{\pi \Delta}+O\left(r^{3 / 2}\right), \quad \mu_{\mathrm{eff}}=r \Delta_{1}+r^{3 / 2} \Delta_{32},
\end{aligned}
$$

where $s_{0}=\sqrt{\mu_{\text {eff }} / m}$ is the sound velocity at $T=0, \Delta_{1}=\Delta$ $\pi /\left(\pi+Q_{0} U m\right), \Delta_{32}=4(\Delta m)^{3 / 2} \sqrt{\pi} U / 3\left(Q_{0} U m+\pi\right)^{5 / 2}$ and $Q_{0}=(6 / \pi)^{1 / 3}$ is the Debye momentum [27]. Then, the low temperature expansion for $(\partial C / \partial T)$ near QCP can be directly obtained as

$$
\left(\frac{\partial C}{\partial T}\right)=-2 m^{2} U^{2}\left(\frac{\partial S}{\partial U}\right)=-\alpha \frac{T^{3}}{r^{3 / 2}}+O\left((T m)^{5}\right),
$$

where $\alpha=m^{9 / 2} U^{2} \pi Q_{0} / 15 \Delta \sqrt{\Delta_{1}}$. It is seen that at low but finite temperatures $(\partial C / \partial T)$ diverges as $1 / r^{3 / 2}$, but there is no divergence at exactly $T=0$, where QPT occurs. Numerical estimations show that $\alpha$ is positive and rather small and hence, at low temperatures the contact decreases very slowly as seen in figure 2(a).

\section{Discussion and conclusion}

We have studied the Tan's contact for spin-gapped quantum magnets, assuming that their low temperature properties are related to that of a triplon gas. To the best of our knowledge, this is the first time the parameter $C$ and its dependence on temperature is investigated for a solid. Naturally, it will be interesting to compare our results with existing ones in the literature. However, there is neither a theoretical nor an experimental study of Tan's contact for $\mu V T$ ensembles at finite temperatures and most of the literature on $C(T)$ concern 1D gases in NVT ensembles.

The temperature dependence of $C$ of harmonically trapped 1D Leib-Liniger Bose gas has been studied by Yao et al [54]. They have found that contact increases at low temperatures, reaches a maximum at $T=T^{*}$ and then starts to decrease, i.e. $\left.(\partial C / \partial T)\right|_{T<T^{*}}>0,\left.(\partial C / \partial T)\right|_{T=T^{*}}=0$ and $\left.(\partial C / \partial T)\right|_{T>T^{*}}<0$. From figures 1 and 2 , one may note that for a $3 \mathrm{D}$ system of bosons the situation is quite the opposite; $\left.(\partial C / \partial T)\right|_{T<T_{\mathrm{c}}}<0$ and $\left.(\partial C / \partial T)\right|_{T>T_{\mathrm{c}}}>0$. But at the critical temperature $T_{\mathrm{c}}$, in both cases $C(T)$ exhibits an extremum as a function of temperature, maximum for 1D bosons and minimum for triplons, i.e. in both cases $(\partial C / \partial T)$, changes its sign near the critical temperature. Bearing in mind the relation $(\partial C / \partial T) \sim(\partial S / \partial U)$, one may conclude that at $T=T_{\mathrm{c}}$ the interaction dependence of the entropy displays a maximum. Note that in the case of $1 \mathrm{D}$ bosons this maximum provides a signature of the crossover to the fermionized regime, while in the case of quantum magnets it corresponds just to the point of finite temperature phase transition, where accumulation of entropy occurs [26].

In the present work the temperature dependence of $C(T)$ at large temperatures $T>T_{\mathrm{c}}$, is found to be almost linear. This is to be compared with the results by Vignolo and Minguzzi [55] who obtained the large temperature behavior as $C \sim \sqrt{T}$ for a 1D Bose gas in the Tonks-Girardeau limit. It would be interesting to study the effect of dimensionality on $C(T)$ in $\mu V T$ ensembles also [56].

We have studied the critical behavior of $C$ at the QPT and found that $C$ and $(\partial C / \partial \mu)$ are regular at QCP which is in good agreement with predictions made by Chen et al [25].

In conclusion it should be underlined that our analytical expressions for Tan's contact of spin gapped compounds are expressed in terms of magnetizations which are directly observable. For example, at very low temperatures, $T \ll T_{\mathrm{c}}$, one evaluates $C$ simply from the expression $C=m^{2} \mu^{2} / \bar{a}^{4}$, where the only parameter, effective mass should be estimated. Note that, in this region contact of a $\mu V T$ ensemble is almost not sensitive to the strength of the boson-boson interaction $U$, while the dependence on $U$ of the contact of an NVT ensemble 
is rather strong even in the classical approximation, when $C$ is given by $C(N V T) \approx 16 \pi^{2} a^{2} \rho^{2}=U^{2} m^{2} \rho^{2}[10,37]$.

Finally, we venture to suggest a way to measure $C$ in quantum magnets, which can be performed in a similar way as it was done in reference [17] (see appendix B for details).

We hope our work will stimulate more studies, especially experimental ones exploring the universality of $\mu V T$ ensembles in quantum magnets. Results presented here will deepen our understanding of the connection between shortranged two-body correlations and magnetic phase transitions in antiferromagnetic materials, which have the potential to be used in developing the next generation of spintronic devices.

\section{Acknowledgments}

This work is supported by the Ministry of Innovative Development of the Republic of Uzbekistan and the Scientific and Technological Research Council of Turkey (TUBITAK) under Grant No. 119N689. AR acknowledges partial support from the Academy of Sciences of the Republic of Uzbekistan. BT acknowledges support from the Turkish Academy of Sciences (TUBA).

\section{Data availability statement}

The data that support the findings of this study are available upon reasonable request from the authors.

\section{Appendix A}

In this appendix we provide explicit expressions for the integrals introduced in equations (3.10), (3.15) and (3.16):

$$
\begin{array}{ll}
I_{1}=-\beta \sum_{k} \omega_{k} f_{\mathrm{B}}^{2}\left(\omega_{k}\right) \mathrm{e}^{\beta \omega_{k}}, & I_{2}=-U \beta \sum_{k} f_{\mathrm{B}}^{2}\left(\omega_{k}\right) \mathrm{e}^{\beta \omega_{k}}, \\
I_{3}=\rho_{\mathrm{c}}^{-1} \sum_{k}\left(\frac{\varepsilon_{k} W_{k}}{E_{k}}-\frac{1}{2}\right), & I_{4}=\beta \sum_{k} f_{\mathrm{B}}^{2}\left(\omega_{k}\right) \varepsilon_{k} \mathrm{e}^{\beta \omega_{k}},
\end{array}
$$

where $f_{\mathrm{B}}(x)=1 /\left(\mathrm{e}^{\beta x}-1\right), W_{k}=\left(1 / 2+f_{\mathrm{B}}\left(E_{k}\right)\right), \omega_{k}=\varepsilon_{k}$ $-\mu+2 U \rho$ and $E_{k}^{2}=\varepsilon_{k}\left(\varepsilon_{k}+2 \mu_{\text {eff }}\right)$.

Equation (3.15) includes $\left(\partial \rho_{1} / \partial \mu_{\text {eff }}\right),\left(\partial \sigma / \partial \mu_{\text {eff }}\right)$ and $\left(\partial \mu_{\text {eff }} / \partial U\right)$. The derivatives with respect to $\mu_{\text {eff }}$ can be found by differentiating equations (2.5) to give

$$
\begin{gathered}
\left(\frac{\partial \rho_{1}}{\partial \mu_{\mathrm{eff}}}\right)=\sum_{k} \frac{\varepsilon_{k}}{E_{k}^{2}}\left[\frac{\mu_{\mathrm{eff}} W_{k}}{E_{k}}+\frac{\left(\varepsilon_{k}+\mu_{\mathrm{eff}}\right) W_{k}^{\prime}}{4}\right], \\
\left(\frac{\partial \sigma}{\partial \mu_{\mathrm{eff}}}\right)=-\sum_{k} \frac{\varepsilon_{k}}{E_{k}^{2}}\left[\frac{\mu_{\mathrm{eff}} W_{k}^{\prime}}{4}+\frac{\left(\varepsilon_{k}+\mu_{\mathrm{eff}}\right) W_{k}}{E_{k}}\right],
\end{gathered}
$$

where $W_{k}^{\prime}=\beta\left(1-4 W_{k}^{2}\right)$.

As to $\left(\partial \mu_{\text {eff }} / \partial U\right)$, it can be obtained by differentiation of both sides of the equation $\mu_{\text {eff }}(U)=$ $\mu+2 U\left[\sigma\left(\mu_{\mathrm{eff}}(U)\right)-\rho_{1}\left(\mu_{\mathrm{eff}}(U)\right)\right]$ with respect to $U$ and then solving it for $\left(\partial \mu_{\mathrm{eff}} / \partial U\right)$. This leads to

$$
\begin{array}{r}
\left(\frac{\partial \mu_{\mathrm{eff}}}{\partial U}\right)=\frac{\mu_{\mathrm{eff}}-\mu}{U D}, \\
D=1+2 U\left[\left(\frac{\partial \rho_{1}}{\partial \mu_{\mathrm{eff}}}\right)-\left(\frac{\partial \sigma}{\partial \mu_{\mathrm{eff}}}\right)\right] .
\end{array}
$$

Now, we proceed to evaluate $(\partial C / \partial H)=-2 m^{2} U^{2} g \mu_{\mathrm{B}}$ $(\partial \rho / \partial U)$ and $(\partial C / \partial T)=-2 m^{2} U^{2}(\partial S / \partial U)$ near the critical temperature $T_{\mathrm{c}}$.

From the explicit expression

$$
S\left(T>T_{\mathrm{c}}\right)=\sum_{k} \ln \left(1-\mathrm{e}^{-\beta \omega_{k}}\right)+\beta \sum_{k} \omega_{k} f_{\mathrm{B}}\left(\omega_{k}\right),
$$

where $\omega_{k}=\varepsilon_{k}-\mu+2 U \rho(U)$, one obtains

$$
\left(\frac{\partial S}{\partial U}\right)_{T=T_{\mathrm{c}}+0}=-\beta^{2} \sum_{k} \mathrm{e}^{\beta \omega_{k}} \omega_{k} \omega_{k}^{\prime} f_{\mathrm{B}}^{2}\left(\omega_{k}\right),
$$

where $\omega_{k}^{\prime}=\left(\partial \omega_{k} / \partial U\right)=2 \rho+2 U(\partial \rho / \partial U)$ and $(\partial \rho / \partial U)$ is given by equation (3.10). It is straightforward to show that the integral $I_{2}$ in equation (3.10) is divergent at $T=T_{\mathrm{c}}$, where $\omega_{k}=\varepsilon_{k} \sim k^{2} / 2 m$, and hence $\left.(\partial \rho / \partial U)\right|_{T=T_{\mathrm{c}}}=-\rho / U$. This means that $\omega_{k}^{\prime}\left(T=T_{\mathrm{c}}^{+}\right)=0$ and $\left.(\partial S / \partial U)\right|_{T=T_{\mathrm{c}}^{+}}=0$. Therefore $\left.(\partial C / \partial T)\right|_{T=T_{\mathrm{c}}^{+}}=0$ and $\left.(\partial \rho / \partial U)\right|_{T=T_{\mathrm{c}}^{+}}=-\rho / U$.

Replacing $\omega_{k}$ by $E_{k}$ in equation (A.6) and taking the derivative one finds

$$
\left(\frac{\partial S}{\partial U}\right)_{T<T_{\mathrm{c}}}=-\beta^{2}\left(\frac{\partial \mu_{\mathrm{eff}}}{\partial U}\right) \sum_{k} \varepsilon_{k} \mathrm{e}^{\beta E_{k}} f_{\mathrm{B}}^{2}\left(E_{k}\right) .
$$

Now, taking into account that $\mu_{\text {eff }}\left(T_{\mathrm{c}}\right)=0, \rho\left(T_{\mathrm{c}}\right)=\rho_{\mathrm{c}}=$ $\mu / 2 U, E_{k}\left(T_{\mathrm{c}}\right)=\varepsilon_{k}$ and using equation (A.5) we obtain

$$
\left(\frac{\partial S}{\partial U}\right)_{T=T_{\mathrm{c}}^{-}}=\frac{\mu \beta^{2}}{U D} \sum_{k} \varepsilon_{k} \mathrm{e}^{\beta} \varepsilon_{k} f_{\mathrm{B}}^{2}\left(\varepsilon_{k}\right)
$$

which proves the equation (3.19). The derivative $\left.(\partial \rho / \partial U)\right|_{T=T_{\mathrm{c}}^{-}}$may be obtained from $\rho(U)=\left(\mu_{\mathrm{eff}}+U\right) / 2 U$ as

$$
\left(\frac{\partial \rho}{\partial U}\right)_{T=T_{\mathrm{c}}^{-}}=-\frac{\mu(1+D)}{2 U^{2} D}
$$

where $D$ is given by equation (A.5) and $\left(\partial \mu_{\text {eff }}\right) /(\partial U)$ should be evaluated at $T=T_{\mathrm{c}}$.

The expansion of entropy and the effective chemical potential near QCP may be found in the appendix of reference [27].

\section{Appendix B}

Here we outline a sketch of a possible method of measuring Tan's contact for spin gapped magnets. We presume that this can be achieved in a similar way to that for atomic gases.

Wild et al [17] measured $C$ for ${ }^{85} \mathrm{Rb}$ atoms in a gas with a $60 \%$ condensate fraction. The atoms are in the $\mid F=2$, $\left.m_{F}=-2\right\rangle$ state, where $F$ is the total atomic spin and $m_{F}$ is 


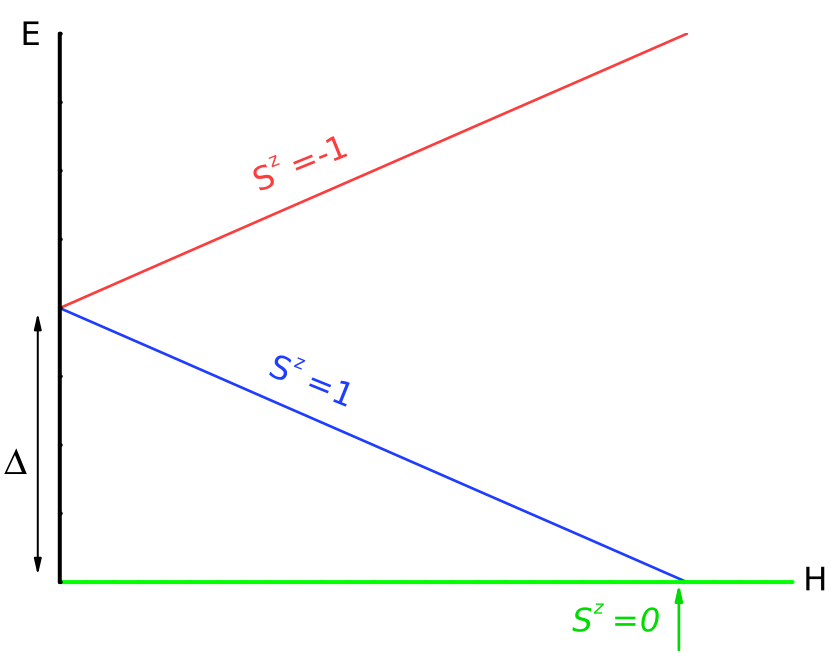

Figure 4. Cartoon of the spin levels of a typical spin gapped quantum magnet showing that the there are $S_{z}= \pm 1$ excited doublet and $S_{z}=0$ ground states. They are separated by a gap $\Delta=g \mu_{\mathrm{B}} H_{\mathrm{c}}$, which can be collapsed due to Zeeman effect for $H>H_{\mathrm{c}}$.

the spin projection. Then by applying an rf pulse the atoms are driven from $|2,-2\rangle$ to $|2,-1\rangle$ state. The rate for transferred atoms to the final spin state is given by $[5,17]$

$$
\lim _{\omega \rightarrow \infty} \Gamma(\omega)=\frac{\Omega^{2}}{4 \pi} F(a, \omega, m) C,
$$

where $\Omega$ is the Rabi frequency, $F(a, \omega, m)$ is a given function of scattering length $a$, the frequency $\omega$, and the atomic mass $m$. Tan's contact has been extracted from this equation after a direct measurement of $\Gamma$ as a function of $\omega$.

Now we consider the spin gapped magnets. Here for $H>H_{\text {c }}$ we have two spin levels with $S_{z}=1$ (ground state) and $S_{z}=-1$ (nearest excited state), as illustrated in figure 4 . The direct transition between the spin singlet and triplet states is, in principle, forbidden in magnetic dipole transitions. Nonetheless, such transitions have been observed in many spin gapped systems by means of high frequency electron spin resonance measurements [57-60]. It was shown that, these transitions are, in fact, driven by ac electric fields, and observed electric dipole transitions can be explained by spin dependent polarization. Very recently, Matsumoto et al [60] developed a theoretical description of singlet-triplet transitions in spin gapped magnet $\mathrm{KCuCl}_{3}$ in the framework of spin-wave theory, and proposed practical formulas for the transition probabilty $W$, using Fermi's golden rule (see e.g. equation (18) of reference [60]). We surmise that if one uses an effective Hamiltonian similar to equation (2.1) the transition probability from reference [60] can be related to the Tan's contact as in equation (B.1), and hence one will be able to obtain $C$ from measurements of $W$, using proper normalization of observed quantities.

\section{ORCID iDs}

Tolib Abdurakhmonov (D) https://orcid.org/0000-0002-50798036

B Tanatar (D) https://orcid.org/0000-0002-5246-0119

\section{References}

[1] Tan S 2008 Energetics of a strongly correlated Fermi gas Ann. Phys., NY 3232952

[2] Tan S 2008 Large momentum part of a strongly correlated Fermi gas Ann. Phys., NY $\mathbf{3 2 3} 2971$

[3] Tan S 2008 Generalized virial theorem and pressure relation for a strongly correlated Fermi gas Ann. Phys., NY 3232987

[4] Braaten E and Platter L 2008 Exact relations for a strongly interacting Fermi gas from the operator product expansion Phys. Rev. Lett. 100205301

[5] Braaten E, Kang D and Platter L 2010 Short-time operator product expansion for rf spectroscopy of a strongly interacting Fermi gas Phys. Rev. Lett. 104223004

[6] Braaten E, Kang D and Platter L 2011 Universal relations for identical bosons from three-body physics Phys. Rev. Lett. 106 153005

[7] Lang G, Vignolo P and Minguzzi A 2017 Tan's contact of a harmonically trapped one-dimensional Bose gas: strongcoupling expansion and conjectural approach at arbitrary interactions Eur. Phys. J. Spec. Top. 2261583

[8] Werner F and Castin Y 2012 General relations for quantum gases in two and three dimensions. II. Bosons and mixtures Phys. Rev. A 86053633

[9] Combescot R, Alzetto F and Leyronas X 2009 Particle distribution tail and related energy formula Phys. Rev. A 79 053640

[10] Pitaevskii L and Stringari S 2015 Bose-Einstein Condensation and Superfluidity (New York: Oxford University Press)

[11] Kuhnle E D, Hoinka S, Dyke P, Hu H, Hannaford P and Vale C J 2011 Temperature dependence of the universal contact parameter in a unitary Fermi gas Phys. Rev. Lett. 106 170402

[12] Sagi Y, Drake T E, Paudel R and Jin D S 2012 Measurement of the homogeneous contact of a unitary Fermi gas Phys. Rev. Lett. 109220402

[13] Stewart J T, Gaebler J P, Drake T E and Jin D S 2010 Verification of universal relations in a strongly interacting Fermi gas Phys. Rev. Lett. 104235301

[14] Chang R, Bouton Q, Cayla H, Qu C, Aspect A, Westbrook C I and Clément D 2016 Momentum-resolved observation of thermal and quantum depletion in a Bose gas Phys. Rev. Lett. 117235303

[15] Fletcher R J, Lopes R, Man J, Navon N, Smith R P, Zwierlein M W and Hadzibabic Z 2017 Two- and three-body contacts in the unitary Bose gas Science 355377

[16] Makotyn P, Klauss C E, Goldberger D L, Cornell E A and Jin D S 2014 Universal dynamics of a degenerate unitary Bose gas Nat. Phys. 10116

[17] Wild R J, Makotyn P, Pino J M, Cornell E A and Jin D S 2012 Measurements of Tan's contact in an atomic Bose-Einstein condensate Phys. Rev. Lett. 108145305

[18] Zou Y-Q, Bakkali-Hassani B, Maury C, Le Cerf É, Nascimbene S, Dalibard J and Beugnon J 2021 Tan's two-body contact across the superfluid transition of a planar Bose gas Nat. Commun. 12760

[19] Hoffman M D, Javernick P D, Loheac A C, Porter W J, Anderson E R and Drut J E 2015 Universality in one-dimensional fermions at finite temperature: density, pressure, compressibility, and contact Phys. Rev. A 91033618

[20] Yu Z, Bruun G M and Baym G 2009 Short-range correlations and entropy in ultracold-atom Fermi gases Phys. Rev. A 80 023615

[21] Matveeva N and Astrakharchik G E 2016 One-dimensional multicomponent Fermi gas in a trap: quantum Monte Carlo study New J. Phys. 18065009

[22] Braaten E and Hammer H-W 2013 Universal relation for the inelastic two-body loss rate J. Phys. B: At. Mol. Opt. Phys. 46215203 
[23] Capuzzi P and Vignolo P 2020 Finite temperature contact for a SU(2) Fermi gas trapped in a 1D harmonic confinement Phys. Rev. A 101013633

[24] Patu O I and Klumper A 2017 Universal Tan relations for quantum gases in one dimension Phys. Rev. A 96063612

[25] Chen Y-Y, Jiang Y-Z, Guan X-W and Zhou Q 2014 Critical behaviours of contact near phase transitions Nat. Commun. 55140

[26] Garst M and Rosch A 2005 Sign change of the Grüneisen parameter and magnetocaloric effect near quantum critical points Phys. Rev. B 72205129

[27] Rakhimov A, Gazizulina A, Narzikulov Z, Schilling A and Sherman E Y 2018 Magnetocaloric effect and Grüneisen parameter of quantum magnets with a spin gap Phys. Rev. B 98144416

[28] Marzolino U $2020 \mu \mathrm{PT}$ statistical ensemble: systems with fluctuating energy, particle number, and volume (arXiv:2011. 05141 )

[29] Sachdev S and Bhatt R N 1990 Bond-operator representation of quantum spins: mean-field theory of frustrated quantum Heisenberg antiferromagnets Phys. Rev. B 419323

[30] Rakhimov A, Khudoyberdiev A, Rani L and Tanatar B 2021 Spin-gapped magnets with weak anisotropies: I. Constraints on the phase of the condensate wave function Ann. Phys., NY 424168361

[31] Zapf V, Jaime M and Batista C D 2014 Bose-Einstein condensation in quantum magnets Rev. Mod. Phys. 86563

[32] Rakhimov A, Nishonov M and Tanatar B 2020 Joule-Thomson temperature of a triplon system of dimerized quantum magnets Phys. Lett. A 384126313

[33] Rakhimov A, Nishonov M, Rani L and Tanatar B 2021 Characteristic temperatures of a triplon system of dimerized quantum magnets Int. J. Mod. Phys. B 352150018

[34] Kumar R and Kumar B 2017 Triplon mean-field analysis of an antiferromagnet with degenerate Shastry-Sutherland ground states J. Phys. Commun. 1055020

[35] Dell'Amore R and Schilling A 2008 Fraction of Bose-Einstein condensed triplons in $\mathrm{TlCuCl}_{3}$ from magnetization data Phys. Rev. B 78224403

[36] Bouchoule I and Dubail J 2020 Breakdown of Tan's relation in lossy one-dimensional Bose gases (arXiv:2011.13250 )

[37] Rakhimov A 2020 Tan's contact as an indicator of completeness and self-consistency of a theory Phys. Rev. A 102063306

[38] Proukakis N P and Jackson B 2008 Finite-temperature models of Bose-Einstein condensation J. Phys. B: At. Mol. Opt. Phys. 41203002

[39] Wang D-S, Hu X-H, Hu J and Liu W M 2010 Quantized quasitwo-dimensional Bose-Einstein condensates with spatially modulated nonlinearity Phys. Rev. A 81025604

[40] Wang D-S, Song S-W, Xiong B and Liu W M 2011 Quantized vortices in a rotating Bose-Einstein condensate with spatiotemporally modulated interaction Phys. Rev. A 84053607

[41] Khudoyberdiev A, Rakhimov A and Schilling A 2017 Bose-Einstein condensation of triplons with a weakly broken U(1) symmetry New J. Phys. 19113002

[42] Giamarchi T and Tsvelik A M 1999 Coupled ladders in a magnetic field Phys. Rev. B 5911398

[43] Grundmann H, Sabitova A, Schilling A, Rohr F. v., Förster T and Peters L 2016 Tuning the critical magnetic field of the triplon Bose-Einstein condensation in $\mathrm{Ba}_{3-x} \mathrm{Sr}_{x} \mathrm{Cr}_{2} \mathrm{O}_{8}$ New J. Phys. 18033001

[44] Misguich G and Oshikawa M 2004 Bose-Einstein condensation of magnons in $\mathrm{TlCuCl}_{3}$ : phase diagram and specific heat from a self-consistent Hartree-Fock calculation with a realistic dispersion relation J. Phys. Soc. Japan 733429

[45] Rakhimov A, Mardonov S and Sherman E Y 2011 Macroscopic properties of triplon Bose-Einstein condensates Ann. Phys., NY 3262499

[46] Rakhimov A, Sherman E Y and Kim C K 2010 High-field instability of a field-induced triplon Bose-Einstein condensate Phys. Rev. B 81020407

[47] Sherman E Y, Lemmens P, Busse B, Oosawa A and Tanaka H 2003 Sound attenuation study on the Bose-Einstein condensation of magnons in $\mathrm{TlCuCl}_{3}$ Phys. Rev. Lett. 91057201

[48] Zhou X-G, Yao Y, Matsuda Y H, Ikeda A, Matsuo A, Kindo $\mathrm{K}$ and Tanaka $\mathrm{H} 2020$ Particle-hole symmetry breaking in a spin-dimer system $\mathrm{TlCuCl}_{3}$ observed at $100 \mathrm{~T}$ Phys. Rev. Lett. 125267207

[49] Rakhimov A, Khudoyberdiev A and Tanatar B 2019 Spingapped magnets with weak anisotropies: II. Effects of exchange and Dzyaloshinsky-Moriya anisotropies on thermodynamic characteristics (arXiv:1909.13641)

[50] Huang K 2001 Introduction to Statistical Physics (Boca Raton, FL: CRC Press)

[51] Rakhimov A and Askerzade I N 2014 Critical temperature of noninteracting bosonic gases in cubic optical lattices at arbitrary integer fillings Phys. Rev. E 90032124

[52] Rakhimov A and Askerzade I N 2015 Thermodynamics of noninteracting bosonic gases in cubic optical lattices versus ideal homogeneous Bose gases Int. J. Mod. Phys. B 291550123

[53] Zhu L, Garst M, Rosch A and Si Q 2003 Universally diverging Grüneisen parameter and the magnetocaloric effect close to quantum critical points Phys. Rev. Lett. 91066404

[54] Yao H, Clément D, Minguzzi A, Vignolo $\mathrm{P}$ and Sanchez-Palencia L 2018 Tan's contact for trapped Lieb-Liniger bosons at finite temperature Phys. Rev. Lett. 121220402

[55] Vignolo P and Minguzzi A 2013 Universal contact for a Tonks-Girardeau gas at finite temperature Phys. Rev. Lett. 110020403

[56] Kosterlitz J M and Thouless D J 1973 Ordering, metastability and phase transitions in two-dimensional systems J. Phys. C: Solid State Phys. 61181

[57] Kimura S, Kindo K and Tanaka H 2004 ESR study of the spin gap system $\mathrm{KCuCl} 3$ using high frequency and high magnetic field Physica B 15346

[58] Kimura S, Matsumoto M, Akaki M, Hagiwara M, Kindo K and Tanaka H 2018 Electric dipole spin resonance in a quantum spin dimer system driven by magnetoelectric coupling Phys. Rev. B 97 140406(R)

[59] Kimura S, Matsumoto M and Tanaka H 2020 Electrical switching of the nonreciprocal directional microwave response in a triplon Bose-Einstein condensate Phys. Rev. Lett. 124 217401

[60] Matsumoto M, Sakurai T, Hirao Y, Ohta H, Uwatoko Y and Tanaka H 2021 First ESR detection of Higgs amplitude mode and analysis with extended spin-wave theory in dimer system $\mathrm{KCuCl}_{3}$ Applied Magnetic Response $\mathbf{5 2} 523$ 